Published in final edited form as:

Curr Ophthalmol Rep. 2018 March ; 6(1): 36-45. doi:10.1007/s40135-018-0161-8.

\title{
Retinal Telemedicine
}

\section{Ru-ik Chee ${ }^{1}$, Dana Darwish ${ }^{1}$, Alvaro Fernandez-Vega, Samir Patel ${ }^{2}$, Karyn Jonas $^{1}$, Susan Ostmo $^{3}$, J. Peter Campbell ${ }^{3}$, Michael F. Chiang ${ }^{3}$, and RV Paul Chan ${ }^{1}$ \\ ${ }^{1}$ Department of Ophthalmology and Visual Sciences, Illinois Eye and Ear Infirmary, University of Illinois at Chicago \\ ${ }^{2}$ Department of Ophthalmology, Wills Eye Hospital, Oregon Health \& Science University, Portland, OR, United States}

${ }^{3}$ Department of Ophthalmology, Casey Eye Institute at Oregon Health \& Science University, Portland, OR, United States

\section{Abstract}

Purpose of Review-An update and overview of the literature on current telemedicine applications in retina.

Recent Findings-The application of telemedicine to the field of Ophthalmology and Retina has been growing with advancing technologies in ophthalmic imaging. Retinal telemedicine has been most commonly applied to diabetic retinopathy and retinopathy of prematurity in adult and pediatric patients respectively. Telemedicine has the potential to alleviate the growing demand for clinical evaluation of retinal diseases. Subsequently, automated image analysis and deep learning systems may facilitate efficient processing of large, increasing numbers of images generated in telemedicine systems. Telemedicine may additionally improve access to education and standardized training through tele-education systems.

Summary-Telemedicine has the potential to be utilized as a useful adjunct but not a complete replacement for physical clinical examinations. Retinal telemedicine programs should be carefully and appropriately integrated into current clinical systems.

\section{Keywords}

Retina; diabetic retinopathy; retinopathy of prematurity; telemedicine; image analysis; deep learning

Corresponding Author: RV Paul Chan, MD, FACS, Department of Ophthalmology and Visual Sciences, Illinois Eye and Ear Infirmary, University of Illinois at Chicago, 1855 W. Taylor Street, Suite 3.138, Chicago, IL, 60612, rvpchan@uic.edu.

Compliance with Ethical Guidelines

Human and Animal Rights and Informed Consent

This article does not contain any studies with human or animal subjects performed by any of the authors.

Conflict of Interest

Ru-ik Chee, Dana Darwish, Alvaro Fernandez-Vega, Samir Patel, Karyn Jonas, Susan Ostmo, Peter Campbell, and R.V. Paul Chan declare no conflict of interest. 


\section{Introduction}

Telemedicine involves the utilization of telecommunication and information technologies for the transfer of clinical data, enabling remote clinical decision-making independent of physical proximity to the patient. The application of telemedicine to the field of Ophthalmology and Retina has been growing with advancing technologies in ophthalmic imaging.[1] Technologies such as ultra-widefield fundus imaging, optical coherence tomography (OCT), pediatric fundus imaging, and non-mydriatic fundus imaging systems allow the efficient acquisition and storage of high quality retinal images. These imaging systems can be effectively operated by trained ancillary healthcare personnel and technicians.[2] Retinal images are subsequently transferred to a centralized reading center, where the data is interpreted remotely and translated into relevant clinical assessments.

Telemedicine may alleviate the overwhelming demand for physical ophthalmic examinations in diseases such as diabetic retinopathy and retinopathy of prematurity (ROP), and expands access to clinical expertise to geographically remote and resource-limited locations. Despite the potential benefits of telemedicine, adoption of such systems requires significant financial and infrastructural investments initially. Nevertheless, telemedicine may prove to be costeffective in the longer term.[3] Implementation of reliable, accurate and dependable new telemedicine systems additionally requires rigorous validation, standardization, and regulation.

The main components required for a retinal telemedicine system include a digital fundus imaging system, image management software, architecture and equipment for image storage and transfer, trained imaging personnel, and ophthalmologists experienced with retinal disease diagnosis and management.

In this review, we discuss the impact of telemedicine and its role in retinal diseases such as diabetic retinopathy and retinopathy of prematurity, as well as ongoing developments in image analysis, deep learning, and tele-education.

\section{Diabetic retinopathy and other adult retinal diseases}

\section{Diabetic retinopathy}

Vision loss from ocular complications associated with diabetes is one of the most common causes of blindness around the world.[4] Given the increasing prevalence of diabetes worldwide, diabetes-related vision loss has the potential to overwhelm the capacity of eye care professionals. Vision loss occurs when complications such as vitreous hemorrhage and retinal detachment develop as a result of uncontrolled diabetic retinopathy, or from diabetic macular edema. Early detection and prompt treatment of ocular complications have been shown to prevent vision loss and blindness in individuals with diabetes.[5, 6]

Early detection of diabetic retinopathy can be achieved through screening, which has traditionally been performed with clinical eye examinations at the physician's office. Screening compliance has been reported to be significantly associated with visual outcomes, [7] yet it has been reported that less than half of the total number of patients with diabetes 
mellitus receive recommended annual screening examinations. [8] Reasons for poor adoption of screening examinations range from lack of patient awareness, delayed referrals from primary care physicians, to various socioeconomic and geographical barriers to care.[9]

Advances in both mydriatic and non-mydriatic fundus imaging systems have facilitated improvements in the ease of acquisition of fundus photographs. Retinal telemedicine may have an important role in increasing the number of patients screened and monitored for diabetic retinopathy. Telemedicine systems do not divert patients away from traditional care settings, but instead increase the number of appropriate eye care referrals.[10] Improved screening rates and less vision loss after implementation of telemedicine programs have also been reported.[11] Importantly, diabetic retinopathy telemedicine screening programs have been shown to be cost effective.[10,12]

The American Telemedicine Association (ATA) classifies diabetic retinopathy telemedicine programs into four categories that vary in the degree of stratification of disease severity. (Table 1) [13] Category 1 programs distinguish no disease, very mild diabetic retinopathy, and greater than mild diabetic retinopathy. Insufficient information is available to make treatment decisions. Examples of category 1 programs include Ophdiat (Paris, France),[14] EyePACS (California, USA),[15] and Digiscope (Baltimore, USA).[16]

Category 2 programs distinguish between vision-threatening (diabetic macular edema, severe nonproliferative diabetic retinopathy, proliferative diabetic retinopathy) and nonvision-threatening disease. Examples of Category 2 programs include Eyecheck (Netherlands)[17] and the National Health Services (NHS) diabetic eye screening program (United Kingdom).[11]

Category 3 programs stratify disease severity into defined levels of mild, moderate, and severe nonproliferative diabetic retinopathy, proliferative diabetic retinopathy, and presence or absence of diabetic macular edema. This level of accuracy and stratification enables appropriate remote decision-making. Examples of Category 3 programs include the Joslin Vision Network (USA)[18] and the University of Alberta (Canada) programs.[19]

Category 4 programs equal or exceed the ability of ETDRS photographs to identify abnormal lesions. There are as yet no validated category 4 telemedicine programs for diabetic retinopathy.

Dilated stereoscopic fundus photographs-In 1999, one of the first major studies evaluating the use of fundus photographs in the detection of diabetic retinopathy was based on fundus photographs from 3711 patients with diabetes enrolled in the Early Treatment Diabetic Retinopathy Study (ETDRS).[5] Multivariate regression techniques were used to identify the characteristics of retinopathy lesions within ETDRS seven standard field 30degree stereo fundus photographs that would form the basis for referral to eye care professionals. Based on a single examination, the authors reported a calculated sensitivity of $87 \%$ for detecting proliferative diabetic retinopathy. Detection of moderate non-proliferative diabetic retinopathy had a sensitivity of $81 \%$ and specificity of $93 \%$. Presence of any hard exudate of moderate or worse severity anywhere in the macular region (ETDRS field 2) had 
an $89 \%$ sensitivity and $58 \%$ specificity for the detection of clinically significant macular edema.

A program utilizing dilated seven-field ETDRS photographs for remote interpretation for diabetic retinopathy was started in 1997 in Alberta, Canada.[19] A large number of patients live over 1000 kilometers from an ophthalmologist in Alberta, and the telemedicine system had successfully screened and recommended referrals to thousands of individuals.

The NHS diabetic eye screening program in the United Kingdom commenced in 2003. In this program England utilizes two 45 degree fields with mydriatic photography on all individuals with diabetes aged 12 and over, while Scotland utilizes one 45 degree field obtained with staged mydriasis, with dilation only if poor quality images are obtained.[11, 20] As a result of the program, it has been suggested that nationwide screening for diabetic retinopathy has been at least partially responsible for the relegation of diabetes related certifiable blindness behind inherited retinal disorders as the most common cause of certifiable blindness amongst working age adults in England and Wales, although many other factors may contribute to such a change.

Although rare, screening systems that utilize dilated fundus photography without prior evaluation of iridocorneal angles may increase the risk of acute angle closure glaucoma attacks.[21] Several telemedicine systems that do not require pharmacological pupil dilation have been shown to be effective, and potentially minimizes the risk of iatrogenic acute angle closure glaucoma attacks.

Non-mydriatic optical fundus photographs-Since 2001, the Joslin Vision Network in the USA has developed several diabetic retinopathy telemedicine screening programs with agencies such as the United States Veterans Administration, Indian Health Service, and other health facilities in Boston, USA and Caracas, Venezuela.[18] The Indian Health Service provides medical care for millions of Native Americans and Alaska Natives across the United States. Initially utilizing a Topcon (Paramus, NJ) TRC-NW6S non-mydriatic retinal camera interfaced with the MegaVision (Santa Barbara, CA) E1-i-RIC camera back, the Joslin Vision Network validated their non-mydriatic technique of obtaining of obtaining fundus photographs (three 45-degree and two 30-degree retinal fields) against the gold standard ETDRS 7-standard fields 35mm stereoscopic color fundus photographs. The group reported substantial agreement between clinical levels of diabetic retinopathy between nonmydriatic images compared to mydriatic ETDRS images $(\mathrm{k}=0.65)$.[22]

The Joslin Vision Network subsequently retrospectively compared non-mydriatic fundus photography with clinical dilated retinal examination by a retina specialist.[23] The group reported that the stage of diabetic retinopathy exactly matched in $72.5 \%$ of cases, was at or within 1 stage level in $89.3 \%$ of patients. Telemedicine referral based on most severe diagnosis in either eye matched clinical examination in $92.5 \%$ of cases, providing evidence that a telemedicine system can compare favorably with clinical practice.

Prospective evaluation of the Joslin Vision Network telemedicine system provided further evidence for telemedicine as a potential alternative to annual retinal examination for patients 
with no or mild diabetic retinopathy and no DME.[24] Telemedicine evaluations for level of diabetic retinopathy exactly matched clinical exam diagnosis in $89.8 \%$ of eyes, was within one level of diabetic retinopathy in all eyes, and exactly matched clinical exam diagnosis for the presence of diabetic macular edema in all eyes.

The Veterans Affairs telemedicine diabetic retinopathy screening program has adopted the Joslin Vision Network protocol.[25] The Veterans Affairs is a federal agency of the USA that provides healthcare services to eligible military veterans at designated medical centers and clinics nationwide, as well as other non-healthcare services. The Veterans Affairs screening program increased the number of patients with known diabetic retinopathy in patients, increased representation of minority populations receiving screening, decreased the overall age of patients who received screening, and distances traveled for screening eye examinations, illustrating the positive impact of a diabetic telemedicine screening system.

Non-mydriatic ultra-widefield retinal imaging-More recent non-mydriatic 200degree ultra-widefield scanning laser ophthalmoscope fundus imaging systems such as Optomap (Optos, plc, Dunfermline, United Kingdom) have facilitated acquisition of fundus areas exceeding that of the ETDRS 7 standard fields, with reduced image acquisition time, while comparing favorably with both dilated ETDRS photography and dilated clinical fundus examination.[26, 27] The scanning laser ophthalmoscope design of the imaging system additionally makes it less susceptible than traditional optical fundus cameras to media opacities.[28] The Joslin Vision Network introduced non-mydriatic ultra-widefield retinal imaging in October 2014, and compared the use of this imaging system against the established non-mydriatic fundus photography system with the optical Topcon TRC-NW6S non-mydriatic retinal camera.[29] The group reported an $81 \%$ reduction of ungradable eyes, 2 -fold increased identification of diabetic retinopathy, and an additional $10 \%$ of patients with peripheral lesions that suggested more severe diabetic retinopathy.[29] These findings provide evidence supporting the use of non-mydriatic ultra-widefield retinal imaging systems in a diabetic retinopathy telemedicine system.

Optical Coherence Tomography-Thus far, telemedicine systems screening for the presence of diabetic macular edema have been based on the detection of retinal edema, or markers for edema such as hard exudates, from two dimensional color fundus photographs. High false positive rates of up to $86.6 \%$ have been reported with use of current diabetic macular edema screening techniques with fundus photographs.[30] Non-mydriatic Spectral Domain Optical Coherence Tomography (SD-OCT) commonly yields high quality three dimensional images of diagnostic value,[31] and has the potential to provide highly sensitive and specific information of the presence or absence of macular edema.[32] A clinical trial evaluating the outcomes of diabetic macular edema identified by SD-OCT utilization at nonophthalmic diabetic care visits has been completed. (ClinicalTrials.gov identifier: NCT01875783) Non-mydriatic SD-OCT may have an important role in the development of an effective telemedicine screening strategy for diabetic macular edema. 


\section{Non-diabetic retinal diseases}

A telemedicine system intended for the screening of diabetic retinopathy was reported to identify non-diabetic ocular abnormalities requiring referral in $25.9 \%$ of patients screened. [23] Other studies have specifically applied telemedical techniques to the screening and monitoring of non-diabetic retinal diseases such as age-related macular degeneration (AMD) and cytomegalovirus (CMV) retinitis. The study on AMD evaluated teleophthalmology screening against retinal-specialist based screening, and reported no significant differences in referral-to-diagnostic imaging and diagnostic imaging-to-treatment times between both groups.[33] The authors report longer wait times to treatment re-initiation when screening for recurrence of neovascular AMD, suggesting that an AMD teleophthalmology system might be better suited to screening purposes, while established AMD patients may be better suited to receiving follow up care in the office with retina specialists. Remote diagnostic screening for CMV retinitis in Northern Thailand achieved a sensitivity of $89-91 \%$ and specificity of $85 \%-88 \%$ amongst image graders.[34] However, a subsequent study in the same region had a mean sensitivity of $30.2 \%$ between three image graders.[35] Small peripheral lesions were noted to be significant contributors to the rate of false negatives. Unlike diabetic retinopathy and AMD that have a significant proportion of disease manifest in the posterior pole, a single peripheral CMV retinitis lesion missed on screening examination may have larger negative consequences to the patient's visual outcome.

\section{Retinopathy of prematurity and other pediatric retinal diseases}

ROP is a vasoproliferative disease of the retina in premature infants that can progress to blindness. Clinical examination with bedside binocular indirect ophthalmoscopy is the established standard for screening, diagnosis and management of ROP. Proficiency in ROP management requires extensive training, which contributes to a shortage of skilled ROP examiners worldwide needed to perform the numerous screening examinations in preterm newborns. Low physician reimbursements, high medico-legal risks, and decentralization of neonatal intensive care units (NICUs) exacerbate the lack ROP experts available.

Furthermore, numbers of preterm newborns at high risk for developing ROP are increasing as a result of further advances in neonatal intensive care management and socioeconomic factors such as increasing rates of teenage pregnancy.

\section{ROP and telemedicine}

ROP as a disease has several characteristics that make it amenable to the application of telemedicine in clinical practice. Diagnostic and management decisions in ROP are made on the clinical appearance of retinal disease, and there is a universally accepted, evidence-based diagnostic classification standard.[36] Retinal appearance can be accurately captured with imaging systems such as the RetCam3 (Natus Medical), PanoCam LT (Visunex Medical Systems), 3nethra neo (Forus Health), and the Icon (Phoenix Clinical) handheld widefield real-time retinal camera. The subsequent use of telemedicine utilizes technology to channel remotely obtained clinical images to a centralized reading center for interpretation, reducing the dependence on physicians' physical proximity to patients and NICUs. Active ROP telemedicine programs in the United States include the Focus-ROP[37] and SUNDROP[38] programs. Internationally, ROP telemedicine programs are ongoing in Canada,[39] 
Germany,[40] New Zealand,[41] and India.[42] ROP telemedicine screening has additionally been shown to be cost-effective in both developed and developing nations.[3, 43]

There have been a number of studies evaluating the effectiveness of telemedicine for ROP, including the Imaging and Informatics in ROP research consortium (i-ROP) and the Telemedicine Approaches to Evaluating Acute-Phase Retinopathy of Prematurity (e-ROP) Study. A 2012 American Academy of Ophthalmology (AAO) Ophthalmic Technology Assessment Committee (OTAC) report on the use of wide-angle digital retinal photography in ROP noted at least five independent level I original studies demonstrating high accuracy (>90\% sensitivity) for the detection of clinically significant ROP.[44] Further level III studies corroborated with reports of high accuracy without detectable complications. The OTAC report concluded that wide-angle digital photography has the potential to complement standard ROP care, as well as provide additional benefits through objective documentation, improved monitoring of disease progression or regression, and as tools for education and research.

A 2015 joint technical report by the American Academy of Pediatrics (AAP) and the American Academy of Ophthalmology further reaffirmed earlier reports of high diagnostic accuracy with the use of wide-angle digital retinal photography for the detection of clinically significant ROP.[45] The joint technical report additionally outlined important practical, pitfalls and risk management considerations with the implementation of an ROP telemedicine system.

Currently, the joint policy statement, which was published in 2013, on the screening for ROP by the AAO, AAP, the American Association of Pediatric Ophthalmology and Strabismus, and the American Association of Certified Orthoptists, does discuss the use of digital photographic retinal images for both documentation and remote interpretation and ROP screening. Although ROP telemedicine may be an acceptable strategy for screening, care must be taken to ensure successful implementation of such a model.

\section{Telemedicine system structure}

The vast majority of infants suitable for inclusion in a retinal telemedicine screening program reside in NICUs and newborn inpatient units within hospitals. Establishing the infrastructure needed in a pediatric retinal telemedicine system differs from that for adult diseases, which requires a focus on different imaging systems and patient locations.

\section{Non-ROP abnormalities and universal newborn screening}

A telemedicine system intended for ROP may uncover other incidental retinal and ocular pathology other than ROP that may be independently clinically significant. Patients with such abnormalities should be identified for repeat imaging or on-site examination. Several pilot studies have additionally evaluated the expansion of a telemedicine screening system to encompass universal newborn screening.[46-49] Universal newborn screening has uncovered new information on the incidence of fundus abnormalities such as fundus hemorrhages.[47, 48] The long-term significance of such findings remains unclear, with many fundus hemorrhages resolving spontaneously within 1-2 weeks without clinically significant visual impairment, and other hemorrhages persisting longer with unknown 
effects.[50] Universal newborn screening has been reported to identify abnormalities such as posterior uveitis, salt and pepper retinopathy, significant retinal hemorrhages, and retinoblastoma, which would not have otherwise been identified from current screening protocols.[47, 48] When factoring in the effects of a lifetime of blindness that may be prevented, universal newborn screening has the potential to be cost effective.[47-49]

\section{Image Analysis}

Recognition of known clinical patterns enable a retinal specialist to analyze and stratify a patient's clinical risk for vision loss based on retina appearance on ophthalmoscopy or fundus images. A computer-based image analysis system similarly analyzes predetermined image characteristics and parameters and generates a customizable output report.

In ROP, determination and intergrader agreement for the subjective presence of plus disease is particularly poor.[51] Computer-based image analysis systems for ROP objectively quantify retinal vascular parameters like arterial tortuosity and venous dilation. Examples of such systems include ROPtool, Retinal Image multiScale Analysis (RISA), Computer-aided Image Analysis of the Retina (CAIAR), VesselMap, and the i-ROP system.[52-54] Computer-based automated image analysis systems for ROP have been reported to be comparable to ROP expert image interpretation in the detection of plus disease.[55-57]

As imaging-based telemedicine systems develop and improve, increasing numbers of fundus photographs need expert review and interpretation. Automating a computer-based image analysis may potentially increase the efficiency of a telemedicine screening system through an initial triage of low risk images, decreasing observer fatigue, and reducing bias.[58]

Automated image analysis begins with segmentation, or identification, of normal anatomy and reference structures such as the optic nerve, fovea and large retinal vessels. Subsequent exclusion of these normal structures allow for analysis of the remaining image and selection detection of predetermined abnormalities such as microaneurysms, hemorrhage, exudates, cotton wool spots, venous beading, and intraretinal microvascular abnormalities. Large ophthalmology data sets such as EyePACS and the Methods for Evaluating Segmentation and Indexing techniques Dedicated to Retinal Ophthalmology (MESSIDOR) database have been used to optimize and validate semi-automated and automated image analysis systems. $[59,60]$

Automated retinal image analysis systems currently in use for the detection of diabetic retinopathy include iGradingM (Medalytix Group Ltd, Scotland, United Kingdom), TRIAD Network (Hubble Telemedical, Inc., Tennessee, USA), Iowa Detection Program (IDx, LLC, Iowa, USA), RetmarkerDR (Retmarker Ltd, Coimbra, Portugal), Retinalyze System (Retinalyze A/S, Horsholm, Denmark), and EyeArt (Eyenuk Inc, Woodland Hills, California, USA). Sensitivity for the detection of referable diabetic retinopathy across the various platforms was upwards of $96 \%$, appropriately high for a use in a screening system. Reported specificities ranged from $52 \%$ to $71 \%$.[58, 60-62] 
The application of automated retinal image analysis to retinal telemedicine systems potentially provide a feasible technique to appropriately triage and manage the significant numbers of images generated by a widely used telemedicine system.

\section{Deep Learning}

Machine learning is the most recent automated computational technology being applied to image analysis in telemedicine systems. It is the latest form of artificial intelligence that when compared to conventional image analysis systems, does not require manual engineering of features to be extracted from raw data, also known as 'feature engineering'. [63] In other words, in the context of retina image analysis, there is no explicit expert input for the identification of representations such as microaneurysms in diabetic retinopathy, or retinal vessel tortuosity in ROP. Instead, the algorithm automatically discovers from within the raw data the representations needed for detection of abnormalities or classification of images into different disease stages.

A deep learning algorithm incorporates multiple levels of representations, transforming the representations at each level of pattern recognition starting from the raw data, all the way to the final complex function. For the purposes of retinal image analysis and classification, particular patterns of pixels represent the presence or absence of edges, particular arrangements of edges represent the presence or absence of motifs, and particular arrangements of motifs correspond to parts of identifiable clinical abnormalities, from which the clinical classification of images may be achieved.

Development of an efficient deep learning system requires a large set of 'training' images, from which the system can be optimized with a backpropagation algorithm.

Backpropagation refers to the backward propagation of errors, which indicates how the machine system should change its internal parameters to compute the representations of each level. With each training image, the severity grade given by the system is compared with the known grade, and the parameters are slightly modified to decrease the error on that image. The training process is then repeated for each image in the set until the system accurately classifies images based on the severity of pathology. The optimized learning system can then be validated against a separate set of images to determine its efficiency, with the ultimate goal to compute severity on new images.

Abramoff et al reported significantly improved performance of automated detection of diabetic retinopathy when deep learning was integrated into the Iowa Detection Program system of automated fundus image analysis.[64] When compared to the Iowa Detection Program without integrated deep learning, sensitivity was not significantly different (95\% confidence interval: $93.3 \%-98.8 \%$ with deep learning vs. $94.4 \%-99.3 \%$ without deep learning), while specificity was significantly improved (95\% confidence interval: $84.2 \%-$ $89.4 \%$ with deep learning vs. 55.7\%-63.0\% without deep learning).

Using large optimization image data sets ( $\mathrm{n}=128,175$ images), Gulshan et al reported high sensitivities and specificities in two separate test image sets. Sensitivity was $97.5 \%$ and specificity was $93.4 \%$ for the detection of referable diabetic retinopathy in the EyePACS 
image set ( $\mathrm{n}=9963$ images), and sensitivity was $96.1 \%$ and specificity was $93.9 \%$ in the MESSIDOR data set ( $n=1748$ images).[65] Gargeya and Leng reported similar high rates of sensitivity (94\%) and specificity (98\%) using a separate deep learning algorithm optimized with 75,137 publicly available fundus images.[66]

Deep learning algorithms have similarly been applied to SD-OCT images in the detection of retinal disease. With optimal cutoffs, Lee et al reported peak sensitivity of $92.64 \%$ and 93.69\% for the detection of AMD.[67] In this study, 80,839 images (41,074 from AMD, 39,765 from normal) were used for optimization, and 20,163 images (11,616 from AMD, 8547 from normal) were used for validation.

Deep learning has recently been applied in ROP and has demonstrated promise for the classification of plus disease using fundus photographs.[68] (Figure 1) There are two potential roles that a deep learning-based classifier could play in ROP screening. First, as part of a telemedicine program, the algorithm could provide a preliminary diagnosis, which would be based on objective features, which could complement the clinical grader's diagnosis to add an element of objectivity. Second, since plus disease presents on a spectrum, the output of the deep learning algorithm could be a continuous severity score, rather than a diagnosis of plus disease. This would have several advantages: (1) a continuous score would objectify the diagnosis of plus disease and allow different observers to compare the same level of disease in clinical care and clinical trials,[68] (2) it could be placed in the clinical context of the overall disease course and risk factors without compelling the clinician to respond to a computer-based diagnosis, [69] and (3) be incorporated into screening programs to identify and refer neonates with clinically significant ROP for ophthalmoscopic exam and treatment.[70] This would further complement the efficiency of human resource utilization with telemedicine by adding an element of objectivity to the diagnosis, reducing human error, a limiting feature of any physician supervised telemedicine system.

A potential limitation of deep learning image analysis systems include the uncertainty of exact "learned" features, as the algorithm may possibly be using patterns unknown to trained ophthalmologists.[71] Further research into the understanding of the patterns used to make predictions by the deep learning function is an active area of research that may not only allow for an opportunity for refinement of the function, but may also provide further understanding of previously unknown manifestations of clinical disease. Additionally, deep learning systems may not be optimized to detect lesions that may not be comprised in the majority of the training image set, leaving possibility of false negatives when applied to new images. Continued learning with real life data may be important to allow for further refinement and optimization of the deep learning function. Furthermore, a deep learning algorithm optimized to identify abnormalities of one particular disease may not be able to identify other abnormal incidental findings that warrant referral. In a telemedicine system dependent on expert grading, other clinical abnormalities may be easily flagged for further evaluation. Incorporating the ability to detect other important clinical abnormalities may be an important step prior to replacement of expert graders in a telemedicine system. Nevertheless, deep learning systems have already shown great potential in the ability to 
efficiently interpret an enormous number of images, which may increase screening rates and improve visual outcomes for particular diseases.

\section{Tele-Education}

Telemedicine systems help overcome geographical and socioeconomic barriers through the use of technological infrastructure. Telemedicine is not limited to the clinical evaluation and provision of care to patients. The Global Education Network for ROP (GEN-ROP) has utilized web-based systems with thousands of validated fundus images to improve education on retinal diseases in the United States and internationally.[72, 73] Such systems have the potential to standardize training through assessment of image grading competency, and is especially important in diseases like ROP, which has a significant shortage of experts, and diabetic retinopathy, which has widespread prevalence. As retinal telemedicine programs become more commonplace, tele-education systems may also be optimized to incorporate a certification process to ensure a consistent level of grading prior to involvement in a telemedicine screening program.

\section{Conclusion}

Clinical diagnosis and management of retinal diseases is heavily dependent on pattern recognition of fundus appearance. Advances in imaging techniques with fundus photography and OCT imaging have facilitated the development of retinal telemedicine systems, where remote acquisition of images containing important clinical information is interpreted in a centralized location. Telemedicine systems have been shown to improve screening rates in a range of adult and pediatric retinal diseases by overcoming geographical and socioeconomic barriers to access. A growing body of evidence has shown that retinal telemedicine systems are reliable and cost-effective. Further developments in computational techniques have led to the development of automated image analysis systems and application of deep learning artificial intelligence algorithms, which further increase the speed and efficiency of telemedicine systems. Telemedical retinal image systems have additionally been shown to be of educational value.

Importantly, telemedicine-based techniques have the potential to significantly reduce but not eliminate the necessity of on-site examinations, especially in individuals who require treatment, or are at high risk for disease progression. In such cases, retinal telemedicine should be utilized as a useful adjunct and not a complete replacement for physical clinical examinations. To maximize the potential benefits, retinal telemedicine programs should be carefully and appropriately integrated into current clinical systems.

\section{Acknowledgments}

Michael Chiang reports grants from National Institutes of Health, the National Science Foundation, unrestricted department funding from Research to Prevent Blindness, personal fees as a consultant for Novartis (Steering Committee member, RAINBOW study), and is an unpaid member of the scientific advisory board for Clarity Medical Systems. 


\section{References}

Papers of particular interest, published recently, have been highlighted as:

- Of importance

•.Of major importance

1. Rathi S, Tsui E, Mehta N, et al. The Current State of Teleophthalmology in the United States. Ophthalmology. 2017; doi: 10.1016/j.ophtha.2017.05.026

2. Lamirel C, Bruce BB, Wright DW, et al. Quality of Nonmydriatic Digital Fundus Photography Obtained by Nurse Practitioners in the Emergency Department: The FOTO-ED Study. Ophthalmology. 2012; 119:617-624. [PubMed: 22218140]

3. Rothschild MI, Russ R, Brennan KA, et al. The Economic Model of Retinopathy of Prematurity (EcROP) Screening and Treatment: Mexico and the United States. Am J Ophthalmol. 2016; 168:110-121. [PubMed: 27130372]

4. Shaw JE, Sicree RA, Zimmet PZ. Global estimates of the prevalence of diabetes for 2010 and 2030. Diabetes Res Clin Pract. 2010; 87:4-14. [PubMed: 19896746]

5. Bresnick GH, Mukamel DB, Dickinson JC, Cole DR. A screening approach to the surveillance of patients with diabetes for the presence of vision-threatening retinopathy. Ophthalmology. 2000; 107:19-24. [PubMed: 10647713]

6. Early photocoagulation for diabetic retinopathy. ETDRS report number 9. Early Treatment Diabetic Retinopathy Study Research Group. Ophthalmology. 1991; 98:766-785. [PubMed: 2062512]

7. Zoega GM, Gunnarsdóttir T, Björnsdóttir S, et al. Screening compliance and visual outcome in diabetes. Acta Ophthalmol Scand. 2005; 83:687-690. [PubMed: 16396645]

8. Lee PP, Feldman ZW, Ostermann J, et al. Longitudinal rates of annual eye examinations of persons with diabetes and chronic eye diseases. Ophthalmology. 2003; 110:1952-1959. [PubMed: 14522771]

9. Zimmer-Galler IE, Kimura AE, Gupta S. Diabetic retinopathy screening and the use of telemedicine. Curr Opin Ophthalmol. 2015; 26:167-172. [PubMed: 25759962]

10. Chasan JE, Delaune B, Maa AY, Lynch MG. Effect of a teleretinal screening program on eye care use and resources. JAMA Ophthalmol. 2014; 132:1045-1051. [PubMed: 24875731]

11. Scanlon PH. The English National Screening Programme for diabetic retinopathy 2003-2016. Acta Diabetol. 2017; 54:515-525. [PubMed: 28224275]

12. Prescott G, Sharp P, Goatman K, et al. Improving the cost-effectiveness of photographic screening for diabetic macular oedema: a prospective, multi-centre, UK study. Br J Ophthalmol. 2014; 98:1042-1049. [PubMed: 24682180]

13. Li HK, Horton M, Bursell S-E, et al. Telehealth practice recommendations for diabetic retinopathy, second edition. Telemed J E Health. 2011; 17:814-837. [PubMed: 21970573]

14. Schulze-Döbold C, Erginay A, Robert N, et al. Ophdiat(®): five-year experience of a telemedical screening programme for diabetic retinopathy in Paris and the surrounding area. Diabetes Metab. 2012; 38:450-457. [PubMed: 22765981]

15. Cuadros J, Bresnick G. EyePACS: an adaptable telemedicine system for diabetic retinopathy screening. J Diabetes Sci Technol. 2009; 3:509-516. [PubMed: 20144289]

16. Zimmer-Galler I, Zeimer R. Results of implementation of the DigiScope for diabetic retinopathy assessment in the primary care environment. Telemed J E Health. 2006; 12:89-98. [PubMed: 16620162]

17. Abramoff MD, Suttorp-Schulten MSA. Web-based screening for diabetic retinopathy in a primary care population: the EyeCheck project. Telemed J E Health. 2005; 11:668-674. [PubMed: 16430386]

18. Sanchez CR, Silva PS, Cavallerano JD, et al. Ocular telemedicine for diabetic retinopathy and the Joslin Vision Network. Semin Ophthalmol. 2010; 25:218-224. [PubMed: 21091003]

19. Ng M, Nathoo N, Rudnisky CJ, Tennant MTS. Improving access to eye care: teleophthalmology in Alberta, Canada. J Diabetes Sci Technol. 2009; 3:289-296. [PubMed: 20144360] 
20. Looker HC, Nyangoma SO, Cromie DT, et al. Rates of referable eye disease in the Scottish National Diabetic Retinopathy Screening Programme. Br J Ophthalmol. 2014; 98:790-795. [PubMed: 24599419]

21. Lagan MA, O'Gallagher MK, Johnston SE, Hart PM. Angle closure glaucoma in the Northern Ireland Diabetic Retinopathy Screening Programme. Eye. 2016; 30:1091-1093. [PubMed: 27229706]

22. Bursell SE, Cavallerano JD, Cavallerano AA, et al. Stereo nonmydriatic digital-video color retinal imaging compared with Early Treatment Diabetic Retinopathy Study seven standard field 35-mm stereo color photos for determining level of diabetic retinopathy. Ophthalmology. 2001; 108:572585. [PubMed: 11237913]

23. Cavallerano AA, Cavallerano JD, Katalinic P, et al. Use of Joslin Vision Network digital-video nonmydriatic retinal imaging to assess diabetic retinopathy in a clinical program. Retina. 2003; 23:215-223. [PubMed: 12707602]

24. Cavallerano JD, Aiello LP, Cavallerano AA, et al. Nonmydriatic digital imaging alternative for annual retinal examination in persons with previously documented no or mild diabetic retinopathy. Am J Ophthalmol. 2005; 140:667-673. [PubMed: 16083842]

25. Kirkizlar E, Serban N, Sisson JA, et al. Evaluation of telemedicine for screening of diabetic retinopathy in the Veterans Health Administration. Ophthalmology. 2013; 120:2604-2610. [PubMed: 24084501]

26. Kernt M, Hadi I, Pinter F, et al. Assessment of diabetic retinopathy using nonmydriatic ultrawidefield scanning laser ophthalmoscopy (Optomap) compared with ETDRS 7-field stereo photography. Diabetes Care. 2012; 35:2459-2463. [PubMed: 22912430]

-27. Silva PS, Cavallerano JD, Sun JK, et al. Nonmydriatic ultrawide field retinal imaging compared with dilated standard 7-field $35-\mathrm{mm}$ photography and retinal specialist examination for evaluation of diabetic retinopathy. Am J Ophthalmol. 2012; 154:549-559.e2. Nonmydriatic ultrawide field images are acquired more rapidly and compare favorably with gold standard dilated ETDRS photography and dilated fundus examination in determining diabetic retinopathy and diabetic macular edema severity. [PubMed: 22626617]

28. Kirkpatrick JN, Manivannan A, Gupta AK, et al. Fundus imaging in patients with cataract: role for a variable wavelength scanning laser ophthalmoscope. Br J Ophthalmol. 1995; 79:892-899. [PubMed: 7488576]

29. Silva PS, Horton MB, Clary D, et al. Identification of Diabetic Retinopathy and Ungradable Image Rate with Ultrawide Field Imaging in a National Teleophthalmology Program. Ophthalmology. 2016; 123:1360-1367. [PubMed: 26949120]

30. Wong RL, Tsang CW, Wong DS, et al. Are we making good use of our public resources? The false-positive rate of screening by fundus photography for diabetic macular oedema. Hong Kong Med J. 2017; 23:356-364. [PubMed: 28684650]

31. Bruce BB, Lamirel C, Biousse V, et al. Feasibility of nonmydriatic ocular fundus photography in the emergency department: Phase I of the FOTO-ED study. Acad Emerg Med. 2011; 18:928-933. [PubMed: 21906202]

32. Ouyang Y, Heussen FM, Keane PA, et al. The retinal disease screening study: retrospective comparison of nonmydriatic fundus photography and three-dimensional optical coherence tomography for detection of retinal irregularities. Invest Ophthalmol Vis Sci. 2013; 54:5694-5700. [PubMed: 23847317]

33. Li B, Powell A-M, Hooper PL, Sheidow TG. Prospective evaluation of teleophthalmology in screening and recurrence monitoring of neovascular age-related macular degeneration: a randomized clinical trial. JAMA Ophthalmol. 2015; 133:276-282. [PubMed: 25473945]

34. Ausayakhun S, Skalet AH, Jirawison C, et al. Accuracy and reliability of telemedicine for diagnosis of cytomegalovirus retinitis. Am J Ophthalmol. 2011; 152:1053-1058.e1. [PubMed: 21861977]

35. Jirawison C, Yen M, Leenasirimakul P, et al. Telemedicine screening for cytomegalovirus retinitis at the point of care for human immunodeficiency virus infection. JAMA Ophthalmol. 2015; 133:198-205. [PubMed: 25412344] 
36. International Committee for the Classification of Retinopathy of Prematurity. The International Classification of Retinopathy of Prematurity revisited. Arch Ophthalmol. 2005; 123:991-999. [PubMed: 16009843]

37. [Accessed 22 Oct 2017] Home Page - FocusROP. http://www.focusrop.com

38. Fijalkowski N, Zheng LL, Henderson MT, et al. Stanford University Network for Diagnosis of Retinopathy of Prematurity (SUNDROP): four-years of screening with telemedicine. Curr Eye Res. 2013; 38:283-291. [PubMed: 23330739]

39. Ells AL, Holmes JM, Astle WF, et al. Telemedicine approach to screening for severe retinopathy of prematurity: a pilot study. Ophthalmology. 2003; 110:2113-2117. [PubMed: 14597517]

40. Lorenz B, Spasovska K, Elflein H, Schneider N. Wide-field digital imaging based telemedicine for screening for acute retinopathy of prematurity (ROP). Six-year results of a multicentre field study. Graefes Arch Clin Exp Ophthalmol. 2009; 247:1251-1262. [PubMed: 19462177]

41. Dai S, Chow K, Vincent A. Efficacy of wide-field digital retinal imaging for retinopathy of prematurity screening. Clin Experiment Ophthalmol. 2011; 39:23-29. [PubMed: 20796264]

42. Vinekar A, Gilbert C, Dogra M, et al. The KIDROP model of combining strategies for providing retinopathy of prematurity screening in underserved areas in India using wide-field imaging, telemedicine, non-physician graders and smart phone reporting. Indian J Ophthalmol. 2014; 62:41-49. [PubMed: 24492500]

43. Castillo-Riquelme MC, Lord J, Moseley MJ, et al. Cost-effectiveness of digital photographic screening for retinopathy of prematurity in the United Kingdom. Int J Technol Assess Health Care. 2004; 20:201-213. [PubMed: 15209180]

-44. Chiang MF, Melia M, Buffenn AN, et al. Detection of clinically significant retinopathy of prematurity using wide-angle digital retinal photography: a report by the American Academy of Ophthalmology. Ophthalmology. 2012; 119:1272-1280. This report by the American Academy of Ophthalmology provides a detailed analysis and evaluation of the quality of evidence of studies related to the detection of clinically significant retinopathy of prematurity with wideangle digital retinal photography. [PubMed: 22541632]

45. Fierson WM, Capone A Jr. American Academy of Pediatrics Section on Ophthalmology, American Academy of Ophthalmology, American Association of Certified Orthoptists. Telemedicine for evaluation of retinopathy of prematurity. Pediatrics. 2015; 135:e238-54. [PubMed: 25548330]

46. Callaway NF, Ludwig CA, Blumenkranz MS, et al. Retinal and Optic Nerve Hemorrhages in the Newborn Infant: One-Year Results of the Newborn Eye Screen Test Study. Ophthalmology. 2016; 123:1043-1052. [PubMed: 26875004]

47. Li L-H, Li N, Zhao J-Y, et al. Findings of perinatal ocular examination performed on 3573, healthy full-term newborns. Br J Ophthalmol. 2013; 97:588-591. [PubMed: 23426739]

48. Vinekar A, Govindaraj I, Jayadev C, et al. Universal ocular screening of 1021 term infants using wide-field digital imaging in a single public hospital in India - a pilot study. Acta Ophthalmol. 2015; 93:e372-6. [PubMed: 25721891]

49. Goyal P, Padhi TR, Das T, et al. Outcome of universal newborn eye screening with wide-field digital retinal image acquisition system: a pilot study. Eye. 2017; doi: 10.1038/eye.2017.129

50. Chee RI, Chan RVP. Universal newborn eye screening: an effective strategy to improve ocular health? Eye. 2017; doi: 10.1038/eye.2017.133

51. Kalpathy-Cramer J, Campbell JP, Erdogmus D, et al. Plus Disease in Retinopathy of Prematurity: Improving Diagnosis by Ranking Disease Severity and Using Quantitative Image Analysis. Ophthalmology. 2016; 123:2345-2351. [PubMed: 27566853]

52. Wittenberg LA, Jonsson NJ, Chan RVP, Chiang MF. Computer-based image analysis for plus disease diagnosis in retinopathy of prematurity. J Pediatr Ophthalmol Strabismus. 2012; 49:11-9. quiz 10-20. [PubMed: 21366159]

53. Campbell JP, Kalpathy-Cramer J, Erdogmus D, et al. Plus Disease in Retinopathy of Prematurity: A Continuous Spectrum of Vascular Abnormality as a Basis of Diagnostic Variability. Ophthalmology. 2016; 123:2338-2344. [PubMed: 27591053]

54. Kalpathy-Cramer J, Campbell JP, Erdogmus D, et al. Plus Disease in Retinopathy of Prematurity: Improving Diagnosis by Ranking Disease Severity and Using Quantitative Image Analysis. Ophthalmology. 2016; 123:2345-2351. [PubMed: 27566853] 
55. Campbell JP, Ataer-Cansizoglu E, Bolon-Canedo V, et al. Expert Diagnosis of Plus Disease in Retinopathy of Prematurity From Computer-Based Image Analysis. JAMA Ophthalmol. 2016; 134:651-657. [PubMed: 27077667]

56. Ataer-Cansizoglu E, Bolon-Canedo V, Peter Campbell J, et al. Computer-Based Image Analysis for Plus Disease Diagnosis in Retinopathy of Prematurity: Performance of the "i-ROP" System and Image Features Associated With Expert Diagnosis. Transl Vis Sci Technol. 2015; 4:5.

57. Abbey AM, Besirli CG, Musch DC, et al. Evaluation of Screening for Retinopathy of Prematurity by ROPtool or a Lay Reader. Ophthalmology. 2016; 123:385-390. [PubMed: 26681393]

58. Sim DA, Keane PA, Tufail A, et al. Automated retinal image analysis for diabetic retinopathy in telemedicine. Curr Diab Rep. 2015; 15:14. [PubMed: 25697773]

59. Philip S, Fleming AD, Goatman KA, et al. The efficacy of automated "disease/no disease" grading for diabetic retinopathy in a systematic screening programme. Br J Ophthalmol. 2007; 91:15121517. [PubMed: 17504851]

60. Abràmoff MD, Folk JC, Han DP, et al. Automated analysis of retinal images for detection of referable diabetic retinopathy. JAMA Ophthalmol. 2013; 131:351-357. [PubMed: 23494039]

61. Tufail A, Rudisill C, Egan C, et al. Automated Diabetic Retinopathy Image Assessment Software: Diagnostic Accuracy and Cost-Effectiveness Compared with Human Graders. Ophthalmology. 2017; 124:343-351. [PubMed: 28024825]

62. Tufail A, Kapetanakis VV, Salas-Vega S, et al. An observational study to assess if automated diabetic retinopathy image assessment software can replace one or more steps of manual imaging grading and to determine their cost-effectiveness. Health Technol Assess. 2016; 20:1-72.

63. LeCun Y, Bengio Y, Hinton G. Deep learning. Nature. 2015; 521:436-444. [PubMed: 26017442]

64. Abràmoff MD, Lou Y, Erginay A, et al. Improved Automated Detection of Diabetic Retinopathy on a Publicly Available Dataset Through Integration of Deep Learning. Invest Ophthalmol Vis Sci. 2016; 57:5200-5206. [PubMed: 27701631]

-65. Gulshan V, Peng L, Coram M, et al. Development and Validation of a Deep Learning Algorithm for Detection of Diabetic Retinopathy in Retinal Fundus Photographs. JAMA. 2016; 316:24022410. "In this evaluation of retinal fundus photographs from adults with diabetes, an algorithm based on deep machine learning had high sensitivity and specificity for detecting referable diabetic retinopathy". [PubMed: 27898976]

66. Gargeya R, Leng T. Automated Identification of Diabetic Retinopathy Using Deep Learning. Ophthalmology. 2017; 124:962-969. [PubMed: 28359545]

67. Lee CS, Baughman DM, Lee AY. Deep Learning Is Effective for Classifying Normal versus AgeRelated Macular Degeneration OCT Images. Ophthalmology Retina. 2017; 1:322-327.

68. Kalpathy-Cramer J, Peter Campbell J, Kim S, et al. Deep learning for the identification of plus disease in retinopathy of prematurity. Invest Ophthalmol Vis Sci. 2017; 58:5554-5554.

69. Peter Campbell J, Kim S, Swan R, et al. Is there clinical utility for a continuous severity score for plus disease in ROP? Invest Ophthalmol Vis Sci. 2017; 58:4737-4737. [PubMed: 28973321]

70. Tibrewal S, Tian P, Kedarisetti D, et al. Evaluation of computer-based image analysis for retinopathy of prematurity screening. Invest Ophthalmol Vis Sci. 2017; 58:5539-5539.

71. Wong TY, Bressler NM. Artificial Intelligence With Deep Learning Technology Looks Into Diabetic Retinopathy Screening. JAMA. 2016; 316:2366. [PubMed: 27898977]

72. Campbell JP, Swan R, Jonas K, et al. Implementation and evaluation of a tele-education system for the diagnosis of ophthalmic disease by international trainees. AMIA Annu Symp Proc. 2015; 2015:366-375. [PubMed: 26958168]

73. Chan RVP, Patel SN, Ryan MC, et al. The Global Education Network for Retinopathy of Prematurity (Gen-Rop): Development, Implementation, and Evaluation of A Novel Tele-Education System (An American Ophthalmological Society Thesis). Trans Am Ophthalmol Soc. 2015; 113:T2. [PubMed: 26538772] 


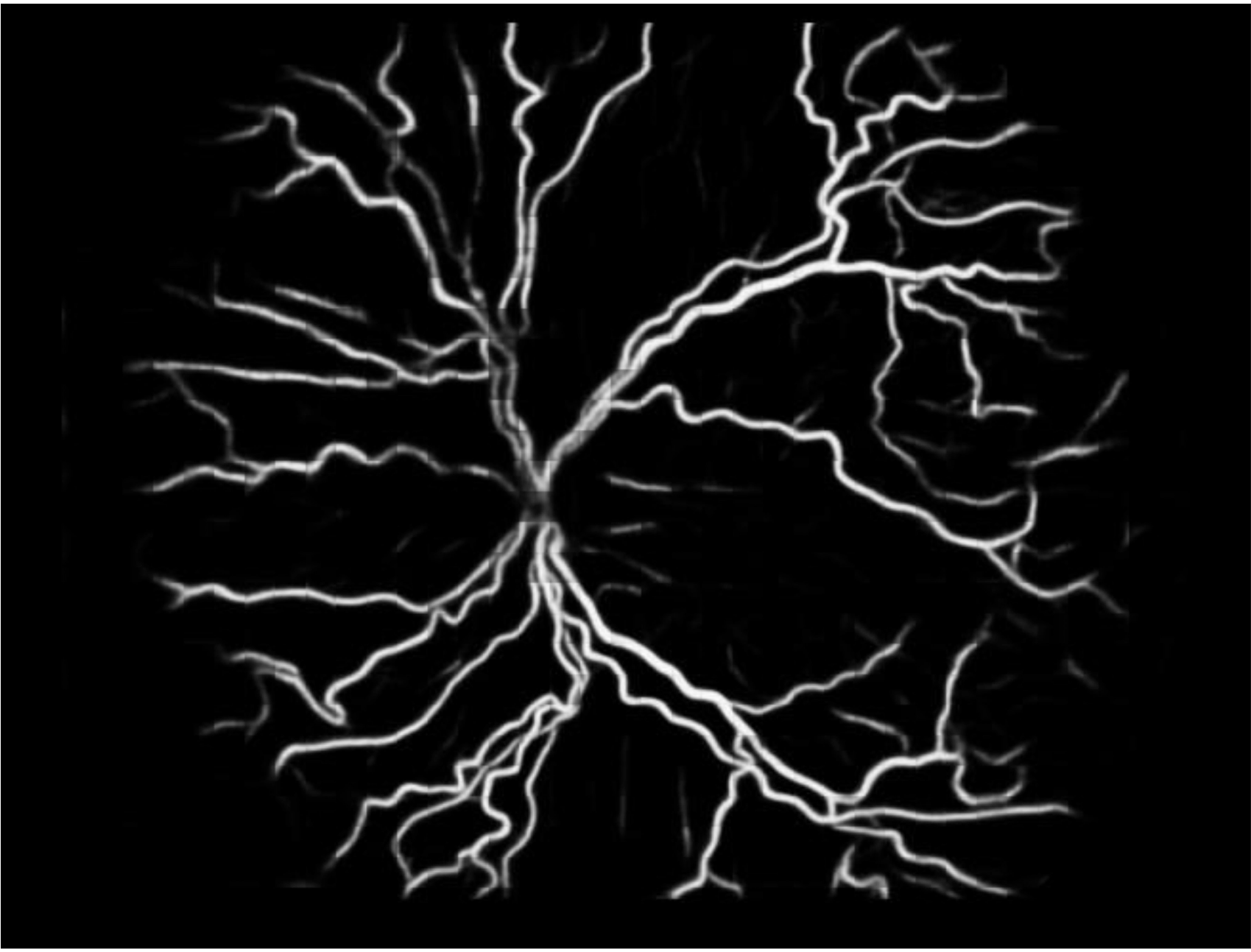

Figure 1.

Image of plus disease processed by the Imaging and Informatics in Retinopathy of Prematurity (i-ROP) deep learning algorithm. 


\section{Table 1}

The American Telemedicine Association (ATA) classification of diabetic retinopathy

\begin{tabular}{|c|c|c|}
\hline CATEGORIES & $\begin{array}{l}\text { STRATIFICATION OF } \\
\text { DISEASE SEVERITY }\end{array}$ & PROGRAMS \\
\hline \multirow{3}{*}{ Category 1} & \multirow{3}{*}{ No DR vs. very mild DR vs. greater severity than mild DR } & Ophdiat ${ }^{14}$ (Paris) \\
\hline & & EyePACS $^{15}$ (California) \\
\hline & & Digiscope $^{16}$ (Baltimore) \\
\hline Category 2 & $\begin{array}{l}\text { Vision-threatening disease (DME, severe NPDR, PDR) vs. Non-vision- } \\
\text { threatening disease }\end{array}$ & $\begin{array}{c}\text { Eyecheck }^{17} \text { (Netherlands) } \\
\text { NHS diabetic eye screening program }{ }^{11} \\
\text { (UK) }\end{array}$ \\
\hline \multirow{2}{*}{ Category 3} & \multirow{2}{*}{$\begin{array}{l}\text { No DR vs. mild NPDR vs. moderate NPDR vs. severe NPDR vs. PDR, and } \\
\text { presence or absence of DME }\end{array}$} & Joslin Vision Network ${ }^{18}$ (USA) \\
\hline & & University of Alberta ${ }^{19}$ (Canada) \\
\hline Category 4 & $\begin{array}{c}\text { Equals or exceeds the ability of ETDRS photographs to determine levels of DR } \\
\text { and DME }\end{array}$ & No validated programs for $D R$ \\
\hline
\end{tabular}

telemedicine programs, and examples of telemedicine programs within each category.

Abbreviations - DR: Diabetic retinopathy. DME: Diabetic macular edema. NPDR: Non-proliferative diabetic retinopathy. PDR: Proliferative diabetic retinopathy. ETDRS: Early Treatment Diabetic Retinopathy Study 